\title{
On the decay and blow-up of solution for a system of nonlinear viscoelastic plate equations with dissipative terms
}

\author{
Jianghao Hao ${ }^{*}$ and Xiaoling Zhang
}

\section{"Correspondence:} hjhao@sxu.edu.cn School of Mathematical Sciences, Shanxi University, Taiyuan, Shanxi 030006, P.R. China

\begin{abstract}
In this paper, we consider the initial-boundary value problem of nonlinear viscoelastic plate equations with dissipative terms. We prove that, for certain initial data in the stable set, the decay rate estimate of the energy function is exponential or polynomial depending on the exponents of the damping terms in both equations by using Nakao's method. Conversely, for certain initial data in the unstable set, we use the perturbed energy method to show that the solution blows up in finite time when the initial energy is not larger than some positive number. This improves earlier results in the literature.
\end{abstract}

MSC: $35 \mathrm{~L} 70 ; 35 \mathrm{~L} 75 ; 93 \mathrm{D} 20$

Keywords: nonlinear plate equations; viscoelastic terms; dissipative terms; decay; blow-up

\section{Introduction}

In this paper, we consider the following initial-boundary value problem of the nonlinear viscoelastic plate equations with dissipative terms:

$$
\left\{\begin{array}{l}
u_{t t}-\gamma \Delta u-\Delta u_{t}+\Delta^{2} u-\Delta u_{t t}+\int_{0}^{t} g_{1}(t-s) \Delta u(s) d s+\left|u_{t}\right|^{p-1} u_{t} \\
\quad=f_{1}(u, v), \quad(x, t) \in \Omega \times(0, T), \\
v_{t t}-\delta \Delta v-\Delta v_{t}+\Delta^{2} v-\Delta v_{t t}+\int_{0}^{t} g_{2}(t-s) \Delta v(s) d s+\left|v_{t}\right|^{q-1} v_{t} \\
\quad=f_{2}(u, v), \quad(x, t) \in \Omega \times(0, T), \\
u(x, 0)=u_{0}(x), \quad u_{t}(x, 0)=u_{1}(x), \quad x \in \Omega, \\
v(x, 0)=v_{0}(x), \quad v_{t}(x, 0)=v_{1}(x), \quad x \in \Omega, \\
u(x, t)=\partial_{v} u(x, t)=0, \quad v(x, t)=\partial_{v} v(x, t)=0, \quad(x, t) \in \partial \Omega \times(0, T),
\end{array}\right.
$$

where $\Omega$ is a bounded domain in $R^{n}(n=1,2,3)$ with smooth boundary $\partial \Omega, \gamma$ and $\delta$ are positive constants, $g_{i}: R^{+} \rightarrow R^{+}, f_{i}: R^{2} \rightarrow R, i=1,2$, are given functions to be specified later.

The motivation of our work is due to the initial boundary problem of the plate equation

$$
\left\{\begin{array}{l}
u_{t t}-\Delta u-\Delta u_{t}+\Delta^{2} u-\Delta u_{t t}+a\left|u_{t}\right|^{m-2} u_{t}=b|u|^{p-2} u, \quad(x, t) \in \Omega \times(0, \infty) \\
u(x, 0)=u_{0}(x), \quad u_{t}(x, 0)=u_{1}(x), \quad x \in \Omega \\
u(x, t)=\partial_{\nu} u(x, t)=0, \quad(x, t) \in \partial \Omega \times(0, T)
\end{array}\right.
$$

\section{Springer}


which has been discussed by Di and Shang [1] by considering the existence of global solutions and the asymptotic behavior of global solutions with $m \geq p$. Here, we understand $\Delta u_{t},-\Delta u_{t t}, a\left|u_{t}\right|^{m-2} u_{t}$, and $b|u|^{p-2} u$ to be the strong dissipation term, the dispersive term, the nonlinear damping term, and the source term, respectively.

In the absence of the dispersive term and the nonlinear damping term, model (1.2) reduces to the following wave equation $(n \geq 1)$

$$
u_{t t}-\Delta u-\Delta u_{t}-\Delta u_{t t}=f(u)
$$

In 2000, Shang [2] studied the well-posedness, asymptotic behavior, and the finite time blow-up of the solutions under some suitable conditions on $f$ and for $n=1,2,3$. In 2004, Zhang and $\mathrm{Hu}$ [3] showed the existence and the stability of global weak solutions. In 2007, Xie and Zhong [4] obtained the existence of global attractors in $H_{0}^{1}(\Omega) \times H_{0}^{1}(\Omega)$, where the nonlinear term $f$ satisfies a critical exponential growth assumption. In 2008, Xu et al. [5] used the multiplier method to investigate the asymptotic behavior of solutions for (1.3). Kafini and Messaoudi [6] considered a nonlinear wave equation and obtained a finite-time blow-up result with arbitrary positive initial energy. For more related results, the reader is referred to [7-10].

In the absence of the dispersive term and $m=0$, model (1.2) reduces to the wave equation

$$
u_{t t}-\Delta u-\Delta u_{t}-\Delta u_{t t}+u_{t}=|u|^{p-2} u
$$

$\mathrm{Xu}$ and Yang [11] established a blow-up result for certain solutions of (1.4) with arbitrary positive initial energy, where $1<p<\infty$ if $n=1,2$ and $1<p \leq \frac{n+2}{n-2}$ if $n \geq 3$.

Messaoudi and Mukiawa [12] studied the fourth-order viscoelastic plate equation

$$
u_{t t}+\Delta^{2} u-\int_{0}^{t} g(t-s) \Delta^{2} u(s) d s=0
$$

in the bounded domain $\Omega=(0, \pi) \times(-l, l) \subset R^{2}$ with nontraditional boundary conditions. The authors established the well-posedness of the solution and a decay result.

Another model related to (1.1) is

$$
\begin{cases}u_{t t}-\Delta u+\int_{0}^{t} g_{1}(t-s) \Delta u(s) d s+h_{1}\left(u_{t}\right)=f_{1}(u, v), & (x, t) \in \Omega \times(0, T), \\ v_{t t}-\Delta v+\int_{0}^{t} g_{2}(t-s) \Delta v(s) d s+h_{2}\left(v_{t}\right)=f_{2}(u, v), & (x, t) \in \Omega \times(0, T),\end{cases}
$$

where $\Omega$ is a bounded domain in $R^{n}(n=1,2,3)$ with smooth boundary $\partial \Omega$. For problem (1.5) with $h_{1}\left(u_{t}\right)=-\Delta u_{t}$ and $h_{2}\left(v_{t}\right)=-\Delta v_{t}$, Liang and Gao [13] obtained that the decay estimate of the energy function is exponential with certain initial data in the stable set. On the contrary, a solution with positive initial energy blows up in finite time when the initial data is inside the unstable set. For $h_{1}\left(u_{t}\right)=\left|u_{t}\right|^{m-1} u_{t}$ and $h_{2}\left(v_{t}\right)=\left|v_{t}\right|^{r-1} v_{t}$, Han and Wang [14] showed several results concerned with local existence, global existence, and finitetime blow-up with negative initial energy. The latter blow-up result has been improved by Messaoudi, Said-Houari, and Guesmia $[15,16]$ by studying a larger class of initial data for which the initial energy can take positive values and obtained that the rate of decay of the total energy depends on those of the relaxation functions. Wu [17] considered the 
following problem for $(x, t) \in \Omega \times(0, T)$ :

$$
\left\{\begin{array}{l}
u_{t t}-M\left(\|\nabla u\|_{2}^{2}+\|\nabla v\|_{2}^{2}\right) \Delta u+\int_{0}^{t} g(t-s) \Delta u(s) d s+\left|u_{t}\right|^{p-1} u_{t}=f_{1}(u, v), \\
v_{t t}-M\left(\|\nabla u\|_{2}^{2}+\|\nabla v\|_{2}^{2}\right) \Delta v+\int_{0}^{t} h(t-s) \Delta v(s) d s+\left|v_{t}\right|^{p-1} v_{t}=f_{2}(u, v),
\end{array}\right.
$$

where $\Omega$ is a bounded domain in $R^{n}(n=1,2,3)$ with smooth boundary $\partial \Omega$. He obtained that the decay estimate of the energy function is exponential or polynomial depending on the exponents of the damping terms in both equations, and the blow-up of solution with nonnegative initial energy was established.

Motivated by previous works, it is interesting to study the global existence, uniform decay, and finite time blow-up of solution to problem (1.1). Firstly, we establish that the solution is global in time under certain initial data in the stable set. After that, we show the decay estimate of solutions by Nakao's method [18]. Precisely, we establish that the decay estimate of energy function is exponential or polynomial depending on the parameters $p$ and $q$. Secondly, we study the finite time blow-up of problem (1.1) with $\gamma=\delta=1$. By adopting and modifying the methods used in [15] we prove the blow-up of solutions when the energy is negative or nonnegative and less than the critical value $E_{1}$ (given in (4.3)). In this way, our results allow a wider region for the blow-up results.

The paper is organized as follow. In Section 2, we present preliminaries and some lemmas. In Section 3, the global existence and decay property are derived. Finally, the blow-up results of (1.1) with $\gamma=\delta=1$ are obtained in the case of initial energy being nonnegative.

\section{Preliminaries}

In this section, we give some lemmas and assumptions. We use the standard Lebesgue space $L^{p}(\Omega)$ and Sobolev space $H_{0}^{1}(\Omega)$ with their usual products and norms. We use the embedding $H_{0}^{1}(\Omega) \hookrightarrow L^{p}(\Omega)$ for $2 \leq p \leq \frac{2 n}{n-2}$ if $n \geq 3$ or $2 \leq p$ if $n=1,2$. In this case, the embedding constant is denoted by $c_{*}$, that is,

$$
\|u\|_{p} \leq c_{*}\|\nabla u\|_{2}
$$

Next, we give the assumptions for problem (1.1).

(A1) The relaxation functions $g_{1}(s)$ and $g_{2}(s)$ are of class $C^{1}$, nonnegative and nonincreasing for $s \geq 0$, and satisfy

$$
\gamma-\int_{0}^{\infty} g_{1}(s) d s=l>0, \quad \delta-\int_{0}^{\infty} g_{2}(s) d s=k>0 .
$$

Concerning the functions $f_{1}(u, v)$ and $f_{2}(u, v)$, we take (see [15])

$$
f_{1}(u, v)=(m+1)\left(a|u+v|^{m-1}(u+v)+b|u|^{\frac{m-3}{2}}|v|^{\frac{m+1}{2}} u\right)
$$

and

$$
f_{2}(u, v)=(m+1)\left(a|u+v|^{m-1}(u+v)+b|v|^{\frac{m-3}{2}}|u|^{\frac{m+1}{2}} v\right)
$$

with constants $a, b>0$. We can easily verify that

$$
u f_{1}(u, v)+v f_{2}(u, v)=(m+1) F(u, v), \quad(u, v) \in R^{2},
$$


where

$$
F(u, v)=a|u+v|^{m+1}+2 b|u v|^{\frac{m+1}{2}} .
$$

(A2) For the nonlinearity, we suppose that

$$
\begin{cases}m>1, & n=1,2 \\ 1<m \leq 3, & n=3\end{cases}
$$

and

$$
\begin{cases}p, q \geq 1, & n=1,2 \\ 1 \leq p, q \leq 5, & n=3\end{cases}
$$

As in [15], we still have the following results.

Lemma 2.1 (Sobolev-Poincaré inequality) Let $2 \leq k<+\infty$ and $n \leq 3$. Then there is a con$\operatorname{stant} \tilde{c}=c(\Omega, k)$ such that

$$
\|u\|_{k} \leq \tilde{c}\|\Delta u\|_{2}, \quad u \in H_{0}^{2}(\Omega)
$$

Lemma 2.2 There exist two positive constants $c_{0}$ and $c_{1}$ such that

$$
c_{0}\left(|u|^{m+1}+|v|^{m+1}\right) \leq F(u, v) \leq c_{1}\left(|u|^{m+1}+|v|^{m+1}\right), \quad(u, v) \in R^{2} .
$$

Lemma 2.3 Suppose that (2.4) holds. Then there exists $\eta>0$ such that, for any $(u, v) \in$ $H_{0}^{1}(\Omega) \times H_{0}^{1}(\Omega)$, we have

$$
\|u+v\|_{m+1}^{m+1}+2\|u v\|_{\frac{m+1}{2}}^{\frac{m+1}{2}} \leq \eta\left(l\|\nabla u\|_{2}^{2}+k\|\nabla v\|_{2}^{2}\right)^{\frac{m+1}{2}} .
$$

We also need the following technical lemma.

Lemma 2.4 ([15]) For any $g \in C^{1}$ and $\phi \in H^{1}(0, T)$, we have

$$
-2 \int_{0}^{t} \int_{\Omega} g(t-s) \phi \phi_{t} d x d s=\frac{d}{d t}\left(g \circ \phi-\int_{0}^{t} g(s) d s\|\phi\|_{2}^{2}\right)+g(t)\|\phi\|_{2}^{2}-g^{\prime} \circ \phi,
$$

where

$$
g \circ \phi:=\int_{0}^{t} g(t-s) \int_{\Omega}|\phi(s)-\phi(t)|^{2} d x d s .
$$

Now, we are in a position to state the local existence result to problem (1.1), which can be established by using arguments similar to those in [14]. We omit the proof.

Theorem 2.5 Let $u_{0}, v_{0} \in H_{0}^{2}(\Omega)$ and $u_{1}, v_{1} \in H_{0}^{1}(\Omega)$. Assume that (A1) and (A2) are satisfied. Then there exists a couple solution $(u, v)$ of problem $(1.1)$ such that, for some $T>0$,

$$
\begin{array}{ll}
u \in L^{\infty}\left(0, T ; H_{0}^{2}(\Omega) \cap L^{p+1}(\Omega)\right), & v \in L^{\infty}\left(0, T ; H_{0}^{2}(\Omega) \cap L^{q+1}(\Omega)\right), \\
u_{t} \in L^{\infty}\left(0, T ; H_{0}^{1}(\Omega)\right) \cap L^{p+1}(\Omega), & v_{t} \in L^{\infty}\left(0, T ; H_{0}^{1}(\Omega)\right) \cap L^{q+1}(\Omega) .
\end{array}
$$


We conclude this section by stating Nakao's lemma, which will be used in establishing the decay rate of solutions to problem (1.1).

Lemma 2.6 ([18]) Let $\phi(t)$ be a nonincreasing and nonnegative function on $[0, T], T>1$, such that

$$
\phi^{1+r}(t) \leq \omega_{0}(\phi(t)-\phi(t+1)), \quad t \in[0, T]
$$

where $\omega_{0}>1$ and $r \geq 0$. Then we have, for all $t \in[0, T]$,

(i) if $r=0$, then

$$
\phi(t) \leq \phi(0) e^{-\omega_{1}[t-1]^{+}},
$$

(ii) if $r>0$, then

$$
\phi(t) \leq\left(\phi^{-r}(0)+\omega_{0}^{-1} r[t-1]^{+}\right)^{-\frac{1}{r}},
$$

where $\omega_{1}:=\ln \left(\frac{\omega_{0}}{\omega_{0}-1}\right)$ and $[t-1]^{+}:=\max \{t-1,0\}$.

Remark 2.7 For simplicity, we take $a=b=1$ in (2.2) and (2.3) throughout this paper.

\section{Global existence and energy decay}

In this section, we focus our attention on the global existence and decay rate of the solution to problem (1.1). We first define

$$
\begin{aligned}
I(t):= & \|\Delta u\|_{2}^{2}+\|\Delta v\|_{2}^{2}+\left\|\nabla u_{t}\right\|_{2}^{2}+\left\|\nabla v_{t}\right\|_{2}^{2}+\left(\gamma-\int_{0}^{t} g_{1}(s) d s\right)\|\nabla u\|_{2}^{2} \\
& +\left(\delta-\int_{0}^{t} g_{2}(s) d s\right)\|\nabla v\|_{2}^{2}+g_{1} \circ \nabla u+g_{2} \circ \nabla v-(m+1) \int_{\Omega} F(u, v) d x, \\
J(t):= & \frac{1}{2}\left(\|\Delta u\|_{2}^{2}+\|\Delta v\|_{2}^{2}+\left\|\nabla u_{t}\right\|_{2}^{2}+\left\|\nabla v_{t}\right\|_{2}^{2}+\left(\gamma-\int_{0}^{t} g_{1}(s) d s\right)\|\nabla u\|_{2}^{2}\right. \\
& \left.+\left(\delta-\int_{0}^{t} g_{2}(s) d s\right)\|\nabla v\|_{2}^{2}+g_{1} \circ \nabla u+g_{2} \circ \nabla v\right)-\int_{\Omega} F(u, v) d x,
\end{aligned}
$$

and define the energy function as

$$
E(t):=\frac{1}{2}\left(\left\|u_{t}\right\|_{2}^{2}+\left\|v_{t}\right\|_{2}^{2}\right)+J(t) .
$$

Lemma 3.1 Suppose that (A1) and (2.4) hold. Let $(u, v)$ be the solution of problem (1.1). Then $E(t)$ is a nonincreasing function, that is, for $t \geq 0$,

$$
\begin{aligned}
\frac{d}{d t} E(t)= & -\left\|\nabla u_{t}\right\|_{2}^{2}-\left\|\nabla v_{t}\right\|_{2}^{2}+\frac{1}{2}\left(g_{1}^{\prime} \circ \nabla u\right)+\frac{1}{2}\left(g_{2}^{\prime} \circ \nabla v\right)-\frac{1}{2} g_{1}(t)\|\nabla u\|_{2}^{2} \\
& -\frac{1}{2} g_{2}(t)\|\nabla v\|_{2}^{2}-\left\|u_{t}\right\|_{p+1}^{p+1}-\left\|v_{t}\right\|_{q+1}^{q+1} .
\end{aligned}
$$


Proof Multiplying $(1.1)_{1}$ by $u_{t}$ and $(1.2)_{2}$ by $v_{t}$, integrating over $\Omega$, summing up, and then using integration by parts, we obtain

$$
\begin{aligned}
\frac{d}{d t}[ & \frac{1}{2}\left(\left\|u_{t}\right\|_{2}^{2}+\left\|v_{t}\right\|_{2}^{2}+\gamma\|\nabla u\|_{2}^{2}+\delta\|\nabla v\|_{2}^{2}+\|\Delta u\|_{2}^{2}+\|\Delta v\|_{2}^{2}+\left\|\nabla u_{t}\right\|_{2}^{2}+\left\|\nabla v_{t}\right\|_{2}^{2}\right) \\
& \left.\quad-\int_{\Omega} F(u, v) d x\right] \\
= & -\left\|\nabla u_{t}\right\|_{2}^{2}-\left\|\nabla v_{t}\right\|_{2}^{2}+\int_{0}^{t} \int_{\Omega} g_{1}(t-s) \nabla u(s) \cdot \nabla u_{t} d x d s \\
& +\int_{0}^{t} \int_{\Omega} g_{2}(t-s) \nabla v(s) \cdot \nabla v_{t} d x d s \\
& -\left\|u_{t}\right\|_{p+1}^{p+1}-\left\|v_{t}\right\|_{q+1}^{q+1} .
\end{aligned}
$$

Applying Lemma 2.4 to the third and fourth terms on the right-hand side of this equality, we get (3.4).

Lemma 3.2 Suppose that (A1) and (2.4) hold. Let $(u, v)$ be the solution of problem (1.1). Assume further that $I(0)>0$ and

$$
\alpha_{1}:=(m+1) c_{1} \tilde{c}^{m+1}\left(\frac{2(m+1)}{m-1} E(0)\right)^{\frac{m-1}{2}}<1
$$

Then

$$
I(t)>0, \quad t \geq 0 .
$$

Proof Since $I(0)>0$, by continuity there exists a maximal time $t_{\max }>0$ (possibly $\left.t_{\max }=T\right)$ such that

$$
I(t) \geq 0, \quad t \in\left[0, t_{\max }\right]
$$

which implies that, for $t \in\left[0, t_{\max }\right]$,

$$
\begin{aligned}
J(t)= & \frac{m-1}{2(m+1)}\left(\|\Delta u\|_{2}^{2}+\|\Delta v\|_{2}^{2}+\left\|\nabla u_{t}\right\|_{2}^{2}+\left\|\nabla v_{t}\right\|_{2}^{2}+\left(\gamma-\int_{0}^{t} g_{1}(s) d s\right)\|\nabla u\|_{2}^{2}\right. \\
& \left.+\left(\delta-\int_{0}^{t} g_{2}(s) d s\right)\|\nabla v\|_{2}^{2}+g_{1} \circ \nabla u+g_{2} \circ \nabla v\right)+\frac{1}{m+1} I(t) \\
\geq & \frac{m-1}{2(m+1)}\left(\|\Delta u\|_{2}^{2}+\|\Delta v\|_{2}^{2}+l\|\nabla u\|_{2}^{2}+k\|\nabla v\|_{2}^{2}\right) .
\end{aligned}
$$

Since $E(t)$ is nonincreasing by (3.4), using (3.7) and (3.3), we get, for $t \in\left[0, t_{\max }\right]$,

$$
\begin{aligned}
& \|\Delta u\|_{2}^{2}+\|\Delta v\|_{2}^{2}+l\|\nabla u\|_{2}^{2}+k\|\nabla v\|_{2}^{2} \\
& \quad \leq \frac{2(m+1)}{m-1} J(t) \leq \frac{2(m+1)}{m-1} E(t) \leq \frac{2(m+1)}{m-1} E(0) .
\end{aligned}
$$


Using Lemma 2.2, (2.6), (3.8), and (3.5), we obtain, for $t \in\left[0, t_{\max }\right]$,

$$
\begin{aligned}
(m+1) \int_{\Omega} F(u, v) d x & \leq(m+1) c_{1}\left(\|u\|_{m+1}^{m+1}+\|v\|_{m+1}^{m+1}\right) \\
& \leq(m+1) c_{1} \tilde{c}^{m+1}\left(\|\Delta u\|_{2}^{m+1}+\|\Delta v\|_{2}^{m+1}\right) \\
& \leq \alpha_{1}\left(\|\Delta u\|_{2}^{2}+\|\Delta v\|_{2}^{2}\right) \\
& <\|\Delta u\|_{2}^{2}+\|\Delta v\|_{2}^{2}
\end{aligned}
$$

Thus,

$$
I(t)>0, \quad t \in\left[0, t_{\max }\right]
$$

By repeating these steps and using the fact that

$$
\lim _{t \rightarrow t_{\max }}(m+1) c_{1} \tilde{c}^{m+1}\left(\frac{2(m+1)}{m-1} E(t)\right)^{\frac{m-1}{2}} \leq \alpha_{1}<1,
$$

this implies that we can take $t_{\max }=T$.

Lemma 3.3 Let the assumptions of Lemma 3.2 hold. Then there exists $\eta_{1}>1$ such that

$$
\|\Delta u\|_{2}^{2}+\|\Delta v\|_{2}^{2} \leq \eta_{1} I(t), \quad t \in[0, T)
$$

where $\eta_{1}=\frac{1}{1-\alpha_{1}}$.

Proof From (3.9) we have

$$
(m+1) \int_{\Omega} F(u, v) d x \leq \alpha_{1}\left(\|\Delta u\|_{2}^{2}+\|\Delta v\|_{2}^{2}\right) .
$$

Letting $\eta_{1}=\frac{1}{1-\alpha_{1}}$ and using (3.1), we obtain (3.10).

Theorem 3.4 Suppose that (A1) and (A2) hold. Let $u_{0}, v_{0} \in H_{0}^{2}(\Omega)$ and $u_{1}, v_{1} \in H_{0}^{1}(\Omega)$ satisfy $I(0)>0$ and (3.5). Then the solution $(u, v)$ of problem (1.1) is global and bounded. Furthermore, if

$$
\gamma+\delta>\frac{7+5 \eta_{1}}{2} \max \left\{\int_{0}^{\infty} g_{1}(s) d s, \int_{0}^{\infty} g_{2}(s) d s\right\}
$$

then we have the following decay estimates:

(i) if $p=q=1$, then, for $t \geq 0$,

$$
E(t) \leq E(0) e^{-\tau_{1} t}
$$

(ii) if $\max \{p, q\}>1$, then, for $t \geq 0$,

$$
E(t) \leq\left(E^{-\max \left\{\frac{p-1}{2}, \frac{q-1}{2}\right\}}(0)+\tau_{2} \max \left\{\frac{p-1}{2}, \frac{q-1}{2}\right\}[t-1]^{+}\right)^{-\frac{2}{\max \{p, q\}-1}}
$$

where $\tau_{1}$ and $\tau_{2}$ are some positive constants. 
Proof First, to prove that $T=\infty$, it suffices to show that $\|\Delta u\|_{2}^{2}+\|\Delta v\|_{2}^{2}+\left\|\nabla u_{t}\right\|_{2}^{2}+\left\|\nabla v_{t}\right\|_{2}^{2}$ is bounded independently of $t$. Thanks to (3.3), (3.4), and (3.6), we have

$$
\begin{aligned}
E(0) \geq & E(t) \geq J(t) \\
= & \frac{m-1}{2(m+1)}\left(\|\Delta u\|_{2}^{2}+\|\Delta v\|_{2}^{2}+\left\|\nabla u_{t}\right\|_{2}^{2}+\left\|\nabla v_{t}\right\|_{2}^{2}\right. \\
& +\left(\gamma-\int_{0}^{t} g_{1}(s) d s\right)\|\nabla u\|_{2}^{2} \\
& \left.+\left(\delta-\int_{0}^{t} g_{2}(s) d s\right)\|\nabla v\|_{2}^{2}+g_{1} \circ \nabla u+g_{2} \circ \nabla v\right)+\frac{1}{m+1} I(t) \\
> & \frac{m-1}{2(m+1)}\left(\|\Delta u\|_{2}^{2}+\|\Delta v\|_{2}^{2}+\left\|\nabla u_{t}\right\|_{2}^{2}+\left\|\nabla v_{t}\right\|_{2}^{2}\right) .
\end{aligned}
$$

Therefore,

$$
\|\Delta u\|_{2}^{2}+\|\Delta v\|_{2}^{2}+\left\|\nabla u_{t}\right\|_{2}^{2}+\left\|\nabla v_{t}\right\|_{2}^{2} \leq \alpha_{2} E(0),
$$

where $\alpha_{2}$ is a positive constant depending only on $m$. Thus, we obtain the global existence.

We further derive the decay rate of the energy function for problem (1.1) by Nakao's method [18]. For this purpose, we have to show that the energy function defined by (3.3) satisfies the hypothesis of Lemma 2.6. Integrating (3.4) over $[t, t+1]$, we have

$$
E(t)-E(t+1)=D_{1}^{p+1}(t)+D_{2}^{q+1}(t)
$$

where

$$
\begin{aligned}
D_{1}^{p+1}(t):= & \int_{t}^{t+1}\left\|\nabla u_{t}\right\|_{2}^{2} d s-\frac{1}{2} \int_{t}^{t+1} g_{1}^{\prime} \circ \nabla u d s \\
& +\frac{1}{2} \int_{t}^{t+1} g_{1}\|\nabla u\|_{2}^{2} d s+\int_{t}^{t+1}\left\|u_{t}\right\|_{p+1}^{p+1} d s, \\
D_{2}^{q+1}(t):= & \int_{t}^{t+1}\left\|\nabla v_{t}\right\|_{2}^{2} d s-\frac{1}{2} \int_{t}^{t+1} g_{2}^{\prime} \circ \nabla v d s \\
& +\frac{1}{2} \int_{t}^{t+1} g_{2}\|\nabla v\|_{2}^{2} d s+\int_{t}^{t+1}\left\|v_{t}\right\|_{q+1}^{q+1} d s .
\end{aligned}
$$

By (3.13), (3.14), and the Hölder inequality, we observe that

$$
\int_{t}^{t+1} \int_{\Omega}\left|u_{t}\right|^{2} d x d s+\int_{t}^{t+1} \int_{\Omega}\left|v_{t}\right|^{2} d x d s \leq c_{1}(\Omega) D_{1}^{2}(t)+c_{2}(\Omega) D_{2}^{2}(t)
$$

where $c_{1}(\Omega)=\operatorname{vol}(\Omega)^{\frac{p-1}{p+1}}$ and $c_{2}(\Omega)=\operatorname{vol}(\Omega)^{\frac{q-1}{q+1}}$

By the mean value theorem there exist $t_{1} \in\left[t, t+\frac{1}{4}\right]$ and $t_{2} \in\left[t+\frac{3}{4}, t+1\right]$ such that

$$
\left\|u_{t}\left(t_{i}\right)\right\|_{2}^{2}+\left\|v_{t}\left(t_{i}\right)\right\|_{2}^{2} \leq 4 c_{1}(\Omega) D_{1}^{2}(t)+4 c_{2}(\Omega) D_{2}^{2}(t), \quad i=1,2 .
$$


Next, multiplying Eq. (1.1) $)_{1}$ by $u$ and Eq. $(1.1)_{2}$ by $v$, integrating over $\Omega \times\left[t_{1}, t_{2}\right]$, and using integration by parts, we obtain

$$
\begin{aligned}
\int_{t_{1}}^{t_{2}} I(t) d t= & -\int_{t_{1}}^{t_{2}} \int_{\Omega} u u_{t t} d x d t-\int_{t_{1}}^{t_{2}} \int_{\Omega} v v_{t t} d x d t-\int_{t_{1}}^{t_{2}} \int_{\Omega} \nabla u \cdot \nabla u_{t} d x d t \\
& -\int_{t_{1}}^{t_{2}} \int_{\Omega} \nabla v \cdot \nabla v_{t} d x d t-\int_{t_{1}}^{t_{2}} \int_{\Omega} \nabla u \cdot \nabla u_{t t} d x d t \\
& -\int_{t_{1}}^{t_{2}} \int_{\Omega} \nabla v \cdot \nabla v_{t t} d x d t+\int_{t_{1}}^{t_{2}}\left\|\nabla u_{t}\right\|_{2}^{2} d t \\
& +\int_{t_{1}}^{t_{2}}\left\|\nabla v_{t}\right\|_{2}^{2} d t+\int_{t_{1}}^{t_{2}} g_{1} \circ \nabla u d t+\int_{t_{1}}^{t_{2}} g_{2} \circ \nabla v d t \\
& +\int_{t_{1}}^{t_{2}} \int_{\Omega} \int_{0}^{t} g_{1}(t-s) \nabla u(t) \cdot(\nabla u(s)-\nabla u(t)) d s d x d t \\
& +\int_{t_{1}}^{t_{2}} \int_{\Omega} \int_{0}^{t} g_{2}(t-s) \nabla v(t) \cdot(\nabla v(s)-\nabla v(t)) d s d x d t \\
& -\int_{t_{1}}^{t_{2}} \int_{\Omega}\left|u_{t}\right|^{p-1} u_{t} u d x d t-\int_{t_{1}}^{t_{2}} \int_{\Omega}\left|v_{t}\right|^{q-1} v_{t} v d x d t .
\end{aligned}
$$

Integrating by parts and applying the Cauchy-Schwarz inequality in the first term of the right-hand side of (3.17), we obtain

$$
\begin{aligned}
& \left|\int_{t_{1}}^{t_{2}} \int_{\Omega} u u_{t t} d x d t\right| \leq \sum_{i=1}^{2}\left\|u_{t}\left(t_{i}\right)\right\|_{2}\left\|u\left(t_{i}\right)\right\|_{2}+\int_{t_{1}}^{t_{2}}\left\|u_{t}(t)\right\|_{2}^{2} d t, \\
& \left|\int_{t_{1}}^{t_{2}} \int_{\Omega} v v_{t t} d x d t\right| \leq \sum_{i=1}^{2}\left\|v_{t}\left(t_{i}\right)\right\|_{2}\left\|v\left(t_{i}\right)\right\|_{2}+\int_{t_{1}}^{t_{2}}\left\|v_{t}(t)\right\|_{2}^{2} d t, \\
& \left|\int_{t_{1}}^{t_{2}} \int_{\Omega} \nabla u \cdot \nabla u_{t t} d x d t\right| \leq \sum_{i=1}^{2}\left\|\nabla u_{t}\left(t_{i}\right)\right\|_{2}\left\|\nabla u\left(t_{i}\right)\right\|_{2}+\int_{t_{1}}^{t_{2}}\left\|\nabla u_{t}(t)\right\|_{2}^{2} d t,
\end{aligned}
$$

and

$$
\left|\int_{t_{1}}^{t_{2}} \int_{\Omega} \nabla v \cdot \nabla v_{t t} d x d t\right| \leq \sum_{i=1}^{2}\left\|\nabla v_{t}\left(t_{i}\right)\right\|_{2}\left\|\nabla v\left(t_{i}\right)\right\|_{2}+\int_{t_{1}}^{t_{2}}\left\|\nabla v_{t}(t)\right\|_{2}^{2} d t
$$

Now, we estimate the third term of the right-hand side of inequality (3.17). By the CauchySchwarz inequality we have

$$
\left|\int_{t_{1}}^{t_{2}} \int_{\Omega} \nabla u \cdot \nabla u_{t} d x d t\right| \leq \int_{t_{1}}^{t_{2}}\|\nabla u\|_{2}\left\|\nabla u_{t}\right\|_{2} d t
$$

and

$$
\left|\int_{t_{1}}^{t_{2}} \int_{\Omega} \nabla v \cdot \nabla v_{t} d x d t\right| \leq \int_{t_{1}}^{t_{2}}\|\nabla v\|_{2}\left\|\nabla v_{t}\right\|_{2} d t
$$


Since

$$
\begin{aligned}
\int_{\Omega} \int_{0}^{t} g_{1}(t-s) \nabla u(t) \cdot(\nabla u(s)-\nabla u(t)) d s d x \\
=\frac{1}{2}\left[\int_{0}^{t} g_{1}(t-s)\left(\|\nabla u(t)\|_{2}^{2}+\|\nabla u(s)\|_{2}^{2}\right) d s-\int_{0}^{t} g_{1}(t-s)\left(\|\nabla u(t)-\nabla u(s)\|_{2}^{2}\right) d s\right] \\
\quad-\int_{\Omega} \int_{0}^{t} g_{1}(s)|\nabla u(t)|^{2} d s d x \\
=-\frac{1}{2} \int_{\Omega} \int_{0}^{t} g_{1}(s)|\nabla u(t)|^{2} d s d x+\frac{1}{2} \int_{0}^{t} g_{1}(t-s)\|\nabla u(s)\|_{2}^{2} d s-\frac{1}{2}\left(g_{1} \circ \nabla u\right) \\
\leq \frac{1}{2} \int_{0}^{t} g_{1}(t-s)\|\nabla u(s)\|_{2}^{2} d s-\frac{1}{2}\left(g_{1} \circ \nabla u\right)
\end{aligned}
$$

and

$$
\begin{aligned}
& \int_{\Omega} \int_{0}^{t} g_{2}(t-s) \nabla v(t) \cdot(\nabla v(s)-\nabla v(t)) d s d x \\
& \quad \leq \frac{1}{2} \int_{0}^{t} g_{2}(t-s)\|\nabla v(s)\|_{2}^{2} d s-\frac{1}{2}\left(g_{2} \circ \nabla v\right),
\end{aligned}
$$

by (3.18)-(3.25) we have

$$
\begin{aligned}
\int_{t_{1}}^{t_{2}} I(t) d t \leq & \sum_{i=1}^{2}\left\|u_{t}\left(t_{i}\right)\right\|_{2}\left\|u\left(t_{i}\right)\right\|_{2}+\sum_{i=1}^{2}\left\|v_{t}\left(t_{i}\right)\right\|_{2}\left\|v\left(t_{i}\right)\right\|_{2} \\
& +\int_{t_{1}}^{t_{2}}\left(\left\|u_{t}(t)\right\|_{2}^{2}+\left\|v_{t}(t)\right\|_{2}^{2}\right) d t+\int_{t_{1}}^{t_{2}}\|\nabla u\|_{2}\left\|\nabla u_{t}\right\|_{2} d t \\
& +\int_{t_{1}}^{t_{2}}\|\nabla v\|_{2}\left\|\nabla v_{t}\right\|_{2} d t+\sum_{i=1}^{2}\left\|\nabla u_{t}\left(t_{i}\right)\right\|_{2}\left\|\nabla u\left(t_{i}\right)\right\|_{2} \\
& +\sum_{i=1}^{2}\left\|\nabla v_{t}\left(t_{i}\right)\right\|\left\|_{2}\right\| \nabla v\left(t_{i}\right) \|_{2}+2 \int_{t_{1}}^{t_{2}}\left(\left\|\nabla u_{t}\right\|_{2}^{2}+\left\|\nabla v_{t}\right\|_{2}^{2}\right) d t \\
& +\frac{1}{2} \int_{t_{1}}^{t_{2}} \int_{0}^{t} g_{1}(t-s)\|\nabla u(s)\|_{2}^{2} d s d t+\frac{1}{2} \int_{t_{1}}^{t_{2}} \int_{0}^{t} g_{2}(t-s)\|\nabla v(s)\|_{2}^{2} d s d t \\
& +\frac{1}{2} \int_{t_{1}}^{t_{2}} g_{1} \circ \nabla u d t+\frac{1}{2} \int_{t_{1}}^{t_{2}} g_{2} \circ \nabla v d t-\int_{t_{1}}^{t_{2}} \int_{\Omega}\left|u_{t}\right|^{p-1} u_{t} u d x d t \\
& -\int_{t_{1}}^{t_{2}} \int_{\Omega}\left|v_{t}\right|^{q-1} v_{t} v d x d t .
\end{aligned}
$$

Now, we will estimate the right-hand side of (3.26). First, by (3.16), (2.1), and (3.8), letting $\beta=\min \{l, k\}$, we have

$$
\begin{aligned}
\left\|u_{t}\left(t_{i}\right)\right\|_{2}\left\|u\left(t_{i}\right)\right\|_{2} & \leq c_{*} \sqrt{4 c_{1}(\Omega) D_{1}^{2}(t)+4 c_{2}(\Omega) D_{2}^{2}(t)} \sup _{t_{1} \leq s \leq t_{2}}\|\nabla u(s)\|_{2} \\
& \leq c_{*}\left(\frac{2(m+1)}{\beta(m-1)}\right)^{\frac{1}{2}} \sqrt{4 c_{1}(\Omega) D_{1}^{2}(t)+4 c_{2}(\Omega) D_{2}^{2}(t)} \sup _{t_{1} \leq s \leq t_{2}} E^{\frac{1}{2}}(s)
\end{aligned}
$$


and

$$
\left\|v_{t}\left(t_{i}\right)\right\|_{2}\left\|v\left(t_{i}\right)\right\|_{2} \leq c_{*}\left(\frac{2(m+1)}{\beta(m-1)}\right)^{\frac{1}{2}} \sqrt{4 c_{1}(\Omega) D_{1}^{2}(t)+4 c_{2}(\Omega) D_{2}^{2}(t)} \sup _{t_{1} \leq s \leq t_{2}} E^{\frac{1}{2}}(s) .
$$

By the Hölder inequality and (3.13) we find

$$
\int_{t_{1}}^{t_{2}}\left\|\nabla u_{t}\right\|_{2} d t \leq\left(\int_{t_{1}}^{t_{2}} 1^{2} d t\right)^{\frac{1}{2}}\left(\int_{t_{1}}^{t_{2}}\left\|\nabla u_{t}\right\|_{2}^{2} d t\right)^{\frac{1}{2}} \leq D^{\frac{p+1}{2}}(t)
$$

Then we have

$$
\int_{t_{1}}^{t_{2}}\|\nabla u\|_{2}\left\|\nabla u_{t}\right\|_{2} d t \leq\left(\frac{2(m+1)}{\beta(m-1)}\right)^{\frac{1}{2}} D_{1}^{\frac{p+1}{2}}(t) \sup _{t_{1} \leq s \leq t_{2}} E^{\frac{1}{2}}(s)
$$

and similarly we obtain

$$
\int_{t_{1}}^{t_{2}}\|\nabla v\|_{2}\left\|\nabla v_{t}\right\|_{2} d t \leq\left(\frac{2(m+1)}{\beta(m-1)}\right)^{\frac{1}{2}} D_{2}^{\frac{q+1}{2}}(t) \sup _{t_{1} \leq s \leq t_{2}} E^{\frac{1}{2}}(s)
$$

By (3.13) and (3.14) we observe that

$$
\int_{t}^{t+1}\left\|\nabla u_{t}\right\|_{2}^{2} d s+\int_{t}^{t+1}\left\|\nabla v_{t}\right\|_{2}^{2} d s \leq D_{1}^{p+1}(t)+D_{2}^{q+1}(t)
$$

By the mean value theorem there exist $t_{1} \in\left[t, t+\frac{1}{4}\right]$ and $t_{2} \in\left[t+\frac{3}{4}, t+1\right]$ such that

$$
\left\|\nabla u_{t}\left(t_{i}\right)\right\|_{2}^{2}+\left\|\nabla v_{t}\left(t_{i}\right)\right\|_{2}^{2} \leq 4 D_{1}^{p+1}(t)+4 D_{2}^{q+1}(t)
$$

From (3.8) and (3.32) we have

$$
\left\|\nabla u_{t}\left(t_{i}\right)\right\|_{2}\left\|\nabla u\left(t_{i}\right)\right\|_{2} \leq\left(\frac{2(m+1)}{\beta(m-1)}\right)^{\frac{1}{2}} \sqrt{4 D_{1}^{p+1}(t)+4 D_{2}^{q+1}(t)} \sup _{t_{1} \leq s \leq t_{2}} E^{\frac{1}{2}}(s)
$$

and

$$
\left\|\nabla v_{t}\left(t_{i}\right)\right\|_{2}\left\|\nabla v\left(t_{i}\right)\right\|_{2} \leq\left(\frac{2(m+1)}{\beta(m-1)}\right)^{\frac{1}{2}} \sqrt{4 D_{1}^{p+1}(t)+4 D_{2}^{q+1}(t)} \sup _{t_{1} \leq s \leq t_{2}} E^{\frac{1}{2}}(s) .
$$

Applying Young's inequality to convolution $\|\phi * \psi\|_{q} \leq\|\phi\|_{r}\|\psi\|_{s}$ with

$$
\frac{1}{q}=\frac{1}{r}+\frac{1}{s}-1, \quad 1 \leq q, r, s \leq \infty
$$

and noting that if $q=1$, then $r=1$ and $s=1$, we get

$$
\begin{aligned}
\int_{t_{1}}^{t_{2}} \int_{0}^{t} g_{1}(t-s)\|\nabla u(s)\|_{2}^{2} d s d t & \leq \int_{t_{1}}^{t_{2}} g_{1}(t) d t \int_{t_{1}}^{t_{2}}\|\nabla u(t)\|_{2}^{2} d t \\
& \leq(\gamma-\beta) \int_{t_{1}}^{t_{2}}\|\nabla u(t)\|_{2}^{2} d t
\end{aligned}
$$


and

$$
\int_{t_{1}}^{t_{2}} \int_{0}^{t} g_{2}(t-s)\|\nabla v(s)\|_{2}^{2} d s d t \leq(\delta-\beta) \int_{t_{1}}^{t_{2}}\|\nabla v(t)\|_{2}^{2} d t .
$$

From (3.1), (3.9), (3.10), (3.35), (3.36), and (A1) we have

$$
\begin{aligned}
& \frac{1}{2}\left(\int_{t_{1}}^{t_{2}} \int_{0}^{t} g_{1}(t-s)\|\nabla u(s)\|_{2}^{2} d s d t+\int_{t_{1}}^{t_{2}} \int_{0}^{t} g_{2}(t-s)\|\nabla v(s)\|_{2}^{2} d s d t\right) \\
& \quad \leq \frac{\gamma+\delta-\beta}{2 \beta} \int_{t_{1}}^{t_{2}}\left(l\|\nabla u\|_{2}^{2}+k\|\nabla v\|_{2}^{2}\right) d t \\
& \quad \leq \frac{\gamma+\delta-\beta}{2 \beta} \int_{t_{1}}^{t_{2}}\left(I(t)+(m+1) \int_{\Omega} F(u, v) d x\right) d t \\
& \quad \leq \frac{\gamma+\delta-\beta}{2 \beta}\left(1+\eta_{1}\right) \int_{t_{1}}^{t_{2}} I(t) d t
\end{aligned}
$$

To estimate the eleventh and twelfth terms on the right-hand side of (3.26), we use (3.37) to obtain

$$
\begin{aligned}
\frac{1}{2} \int_{t_{1}}^{t_{2}}\left(g_{1} \circ \nabla u+g_{2} \circ \nabla v\right) d t= & \frac{1}{2} \int_{t_{1}}^{t_{2}} \int_{0}^{t} g_{1}(t-s)\left(\|\nabla u(s)-\nabla u(t)\|_{2}^{2}\right) d s d t \\
& +\frac{1}{2} \int_{t_{1}}^{t_{2}} \int_{0}^{t} g_{2}(t-s)\left(\|\nabla v(s)-\nabla v(t)\|_{2}^{2}\right) d s d t \\
\leq & \int_{t_{1}}^{t_{2}} \int_{0}^{t} g_{1}(t-s)\left(\|\nabla u(s)\|_{2}^{2}+\|\nabla u(t)\|_{2}^{2}\right) d s d t \\
& +\int_{t_{1}}^{t_{2}} \int_{0}^{t} g_{2}(t-s)\left(\|\nabla v(s)\|_{2}^{2}+\|\nabla v(t)\|_{2}^{2}\right) d s d t \\
\leq & \frac{2(\gamma+\delta-\beta)}{\beta}\left(1+\eta_{1}\right) \int_{t_{1}}^{t_{2}} I(t) d t .
\end{aligned}
$$

Using Hölder inequality, (2.1), (3.8), and (3.13), we have

$$
\begin{aligned}
\left.\left|\int_{t_{1}}^{t_{2}} \int_{\Omega}\right| u_{t}\right|^{p-1} u_{t} u d x d t \mid & \leq \int_{t_{1}}^{t_{2}}\left\|u_{t}\right\|_{p+1}^{p}\|u\|_{p+1} d t \\
& \leq c_{*} \int_{t_{1}}^{t_{2}}\left\|u_{t}\right\|_{p+1}^{p}\|\nabla u\|_{2} d t \\
& \leq c_{*}\left(\frac{2(m+1)}{\beta(m-1)}\right)^{\frac{1}{2}} \int_{t_{1}}^{t_{2}}\left\|u_{t}\right\|_{p+1}^{p} d t \sup _{t_{1} \leq s \leq t_{2}} E^{\frac{1}{2}}(s) \\
& \leq c_{*}\left(\frac{2(m+1)}{\beta(m-1)}\right)^{\frac{1}{2}} D_{1}^{p}(t) \sup _{t_{1} \leq s \leq t_{2}} E^{\frac{1}{2}}(s)
\end{aligned}
$$

and

$$
\left.\left|\int_{t_{1}}^{t_{2}} \int_{\Omega}\right| v_{t}\right|^{q-1} v_{t} v d x d t \mid \leq c_{*}\left(\frac{2(m+1)}{\beta(m-1)}\right)^{\frac{1}{2}} D_{2}^{q}(t) \sup _{t_{1} \leq s \leq t_{2}} E^{\frac{1}{2}}(s) .
$$


Therefore, applying (3.13)-(3.15), (3.27)-(3.31), (3.33)-(3.34), and (3.37)-(3.40) to (3.26) we obtain

$$
\begin{aligned}
\beta_{2} \int_{t_{1}}^{t_{2}} I(t) d t \leq & 4 c_{*} \beta_{1} \sqrt{4 c_{1}(\Omega) D_{1}^{2}(t)+4 c_{2}(\Omega) D_{2}^{2}(t)} \sup _{t_{1} \leq s \leq t_{2}} E^{\frac{1}{2}}(s) \\
& +c_{1}(\Omega) D_{1}^{2}(t)+c_{2}(\Omega) D_{2}^{2}(t)+\beta_{1}\left(D_{1}^{\frac{p+1}{2}}(t)+D_{2}^{\frac{q+1}{2}}(t)\right) \sup _{t_{1} \leq s \leq t_{2}} E^{\frac{1}{2}}(s) \\
& +2\left(D_{1}^{p+1}(t)+D_{2}^{q+1}(t)\right)+4 \beta_{1} \sqrt{4 D_{1}^{p+1}(t)+4 D_{2}^{q+1}(t)} \sup _{t_{1} \leq s \leq t_{2}} E^{\frac{1}{2}}(s) \\
& +c_{*} \beta_{1}\left(D_{1}^{p}(t)+D_{2}^{q}(t)\right) \sup _{t_{1} \leq s \leq t_{2}} E^{\frac{1}{2}}(s),
\end{aligned}
$$

where

$$
\beta_{1}:=\left(\frac{2(m+1)}{\beta(m-1)}\right)^{\frac{1}{2}}, \quad \beta_{2}:=1-\frac{5(\gamma+\delta-\beta)}{2 \beta}\left(1+\eta_{1}\right) .
$$

Note that the assumption

$$
\gamma+\delta>\frac{7+5 \eta_{1}}{2} \max \left\{\int_{0}^{\infty} g_{1}(s) d s, \int_{0}^{\infty} g_{2}(s) d s\right\}
$$

gives $\beta_{2}>0$. Thus,

$$
\begin{aligned}
\int_{t_{1}}^{t_{2}} I(t) d t \leq & c_{3}\left[\sqrt{4 c_{1}(\Omega) D_{1}^{2}(t)+4 c_{2}(\Omega) D_{2}^{2}(t)} \sup _{t_{1} \leq s \leq t_{2}} E^{\frac{1}{2}}(s)+D_{1}^{2}(t)+D_{2}^{2}(t)\right. \\
& +\left(D_{1}^{\frac{p+1}{2}}(t)+D_{2}^{\frac{q+1}{2}}(t)\right) \sup _{t_{1} \leq s \leq t_{2}} E^{\frac{1}{2}}(s)+D_{1}^{p+1}(t)+D_{2}^{q+1}(t) \\
& +\sqrt{4 D_{1}^{p+1}(t)+4 D_{2}^{q+1}(t)} \sup _{t_{1} \leq s \leq t_{2}} E^{\frac{1}{2}}(s) \\
& \left.+\left(D_{1}^{p}(t)+D_{2}^{q}(t)\right) \sup _{t_{1} \leq s \leq t_{2}} E^{\frac{1}{2}}(s)\right]
\end{aligned}
$$

where

$$
c_{3}:=\frac{\max \left\{4 c_{*} \beta_{1}, c_{1}(\Omega), c_{2}(\Omega), 4 \beta_{1}\right\}}{\beta_{2}} .
$$

On the other hand, from the definition of $J(t)$ and $I(t),(3.9)$, and (3.10) we have

$$
\begin{aligned}
J(t)= & \frac{m-1}{2(m+1)}\left(\|\Delta u\|_{2}^{2}+\|\Delta v\|_{2}^{2}+\left\|\nabla u_{t}\right\|_{2}^{2}+\left\|\nabla v_{t}\right\|_{2}^{2}+\left(\gamma-\int_{0}^{t} g_{1}(s) d s\right)\|\nabla u\|_{2}^{2}\right. \\
& \left.+\left(\delta-\int_{0}^{t} g_{2}(s) d s\right)\|\nabla v\|_{2}^{2}+g_{1} \circ \nabla u+g_{2} \circ \nabla v\right)+\frac{1}{m+1} I(t) \\
= & \frac{m-1}{2(m+1)}\left(I(t)+(m+1) \int_{\Omega} F(u, v) d x\right)+\frac{1}{m+1} I(t) \\
\leq & \left(1+\frac{m-1}{2(m+1)} \eta_{1}\right) I(t) \\
:= & c_{4} I(t) .
\end{aligned}
$$


Hence, integrating (3.3) over $\left(t_{1}, t_{2}\right)$ and then using (3.43), (3.42), and (3.15), we deduce that

$$
\begin{aligned}
\int_{t_{1}}^{t_{2}} E(t) d t= & \frac{1}{2} \int_{t_{1}}^{t_{2}}\left(\left\|u_{t}\right\|_{2}^{2}+\left\|v_{t}\right\|_{2}^{2}\right) d t+\int_{t_{1}}^{t_{2}} J(t) d t \\
\leq & \frac{1}{2} \int_{t_{1}}^{t_{2}}\left(\left\|u_{t}\right\|_{2}^{2}+\left\|v_{t}\right\|_{2}^{2}\right) d t+c_{4} \int_{t_{1}}^{t_{2}} I(t) d t \\
\leq & c_{5}\left[D_{1}^{2}(t)+D_{2}^{2}(t)+\sqrt{4 c_{1}(\Omega) D_{1}^{2}(t)+4 c_{2}(\Omega) D_{2}^{2}(t)} \sup _{t_{1} \leq s \leq t_{2}} E^{\frac{1}{2}}(s)\right. \\
& +\left(D_{1}^{\frac{p+1}{2}}(t)+D_{2}^{\frac{q+1}{2}}(t)\right) \sup _{t_{1} \leq s \leq t_{2}} E^{\frac{1}{2}}(s)+D_{1}^{p+1}(t)+D_{2}^{q+1}(t) \\
& +\sqrt{4 D_{1}^{p+1}(t)+4 D_{2}^{q+1}(t)} \sup _{t_{1} \leq s \leq t_{2}} E^{\frac{1}{2}}(s) \\
& \left.+\left(D_{1}^{p}(t)+D_{2}^{q}(t)\right) \sup _{t_{1} \leq s \leq t_{2}} E^{\frac{1}{2}}(s)\right],
\end{aligned}
$$

where $c_{5}:=c_{3} c_{4}$. By integrating (3.4) over $\left[t, t_{2}\right]$ we obtain

$$
\begin{aligned}
E(t)= & E\left(t_{2}\right)+\int_{t}^{t_{2}}\left(\left\|\nabla u_{t}\right\|_{2}^{2}+\left\|\nabla v_{t}\right\|_{2}^{2}-\frac{1}{2}\left(g_{1}^{\prime} \circ \nabla u\right)-\frac{1}{2}\left(g_{2}^{\prime} \circ \nabla v\right)+\frac{1}{2} g_{1}(s)\|\nabla u\|_{2}^{2}\right. \\
& \left.+\frac{1}{2} g_{2}(s)\|\nabla v\|_{2}^{2}+\left\|u_{t}\right\|_{p+1}^{p+1}+\left\|v_{t}\right\|_{q+1}^{q+1}\right) d s .
\end{aligned}
$$

Since $t_{2}-t_{1} \geq \frac{1}{2}$ and $E(t)$ is nonincreasing in $t$, it follows that

$$
\int_{t_{1}}^{t_{2}} E(t) d t \geq \int_{t_{1}}^{t_{2}} E\left(t_{2}\right) d t \geq \frac{1}{2} E\left(t_{2}\right)
$$

Then, we have

$$
\begin{aligned}
E(t) \leq & 2 \int_{t_{1}}^{t_{2}} E(t) d t+\int_{t}^{t_{2}}\left(\left\|\nabla u_{t}\right\|_{2}^{2}+\left\|\nabla v_{t}\right\|_{2}^{2}\right. \\
& -\frac{1}{2}\left(g_{1}^{\prime} \circ \nabla u\right)-\frac{1}{2}\left(g_{2}^{\prime} \circ \nabla v\right)+\frac{1}{2} g_{1}(s)\|\nabla u\|_{2}^{2} \\
& \left.+\frac{1}{2} g_{2}(s)\|\nabla v\|_{2}^{2}+\left\|u_{t}\right\|_{p+1}^{p+1}+\left\|v_{t}\right\|_{q+1}^{q+1}\right) d s \\
\leq & 2 \int_{t_{1}}^{t_{2}} E(t) d t+D_{1}^{p+1}(t)+D_{2}^{q+1}(t) .
\end{aligned}
$$

Consequently, since $E(t)$ is nonincreasing, combining (3.44) with (3.46), we obtain

$$
\begin{aligned}
E(t) \leq & c_{6}\left(D_{1}^{2}(t)+D_{2}^{2}(t)+\sqrt{4 c_{1}(\Omega) D_{1}^{2}(t)+4 c_{2}(\Omega) D_{2}^{2}(t)} E^{\frac{1}{2}}(t)\right. \\
& +\left(D_{1}^{\frac{p+1}{2}}(t)+D_{2}^{\frac{q+1}{2}}(t)\right) E^{\frac{1}{2}}(t)+D_{1}^{p+1}(t)+D_{2}^{q+1}(t) \\
& \left.+\sqrt{4 D_{1}^{p+1}(t)+4 D_{2}^{q+1}(t)} E^{\frac{1}{2}}(t)+\left(D_{1}^{p}(t)+D_{2}^{q}(t)\right) E^{\frac{1}{2}}(t)\right) .
\end{aligned}
$$


Then a simple application of Young's inequality gives, for $t \geq 0$,

$$
E(t) \leq c_{7}\left[D_{1}^{2}(t)+D_{2}^{2}(t)+D_{1}^{p+1}(t)+D_{2}^{q+1}(t)+D_{1}^{2 p}(t)+D_{2}^{2 q}(t)\right]
$$

where $c_{6}$ and $c_{7}$ are positive constants.

Therefore, we have the following decay estimate.

(i) For $p=q=1$, from (3.47) and (3.12) we get

$$
E(t) \leq c_{8}[E(t)-E(t+1)]
$$

where we choose $c_{8}>1$. Thus, by Lemma 2.6 we obtain

$$
E(t) \leq E(0) e^{-\tau_{1} t}, \quad t \geq 0,
$$

where $\tau_{1}:=\ln \frac{c_{8}}{c_{8}-1}$.

(ii) For $\max \{p, q\}>1$, it follows from (3.47) that, for $t \geq 0$,

$$
E(t) \leq c_{7}\left[\left(1+D_{1}^{p-1}(t)+D_{1}^{2 p-2}(t)\right) D_{1}^{2}(t)+\left(1+D_{2}^{q-1}(t)+D_{2}^{2 q-2}(t)\right) D_{2}^{2}(t)\right] .
$$

Since $D_{1}(t) \leq E^{\frac{1}{p+1}}(t) \leq E^{\frac{1}{p+1}}(0)$ and $D_{2}(t) \leq E^{\frac{1}{q+1}}(t) \leq E^{\frac{1}{q+1}}(0)$ by (3.12) and (3.4), we have, for $t \geq 0$

$$
\begin{aligned}
E(t) & \leq c_{7}\left[\left(1+E^{\frac{p-1}{p+1}}(0)+E^{\frac{2 p-2}{p+1}}(0)\right) D_{1}^{2}(t)+\left(1+E^{\frac{q-1}{q+1}}(0)+E^{\frac{2 q-2}{q+1}}(0)\right) D_{2}^{2}(t)\right] \\
& \leq c_{9}\left(D_{1}^{2}(t)+D_{2}^{2}(t)\right) .
\end{aligned}
$$

Then, setting $\rho:=\max \left\{\frac{p-1}{2}, \frac{q-1}{2}\right\}$, we obtain

$$
\begin{aligned}
E^{1+\rho}(t) & \leq\left[c_{9}\left(D_{1}^{2}(t)+D_{2}^{2}(t)\right)\right]^{1+\rho} \\
& \leq c_{10}\left(D_{1}^{2 \rho+2}(t)+D_{2}^{2 \rho+2}(t)\right) \\
& =c_{10}\left(D_{1}^{2 \rho-p+1}(t) D_{1}^{p+1}(t)+D_{2}^{2 \rho-q+1}(t) D_{2}^{q+1}(t)\right) \\
& \leq c_{10}\left(E^{\frac{2 \rho-p+1}{p+1}}(0) D_{1}^{p+1}(t)+E^{\frac{2 \rho-q+1}{q+1}}(0) D_{2}^{q+1}(t)\right) \\
& \leq c_{11}\left(D_{1}^{p+1}(t)+D_{2}^{q+1}(t)\right) \\
& =c_{11}(E(t)-E(t+1)),
\end{aligned}
$$

where $c_{10}:=2^{\rho} \cdot c_{9}^{1+\rho}$ and $c_{11}:=c_{10} \max \left(E^{\frac{2 \rho-p+1}{p+1}}(0), E^{\frac{2 \rho-q+1}{q+1}}(0)\right)$. Application of Lemma 2.6 to (3.48) yields

$$
E(t) \leq\left(E^{-\rho}(0)+\tau_{2} \rho[t-1]^{+}\right)^{-\frac{1}{\rho}}, \quad t \geq 0,
$$

with $\tau_{2}:=c_{11}^{-1}$.

The proof of Theorem 3.4 is completed. 


\section{Blow-up result}

In this section, we deal with the blow-up of solution to problem (1.1) with $\gamma=\delta=1$. In order to state our result, we make an extra assumption on $g_{1}$ and $g_{2}$,

$$
\max \left\{\int_{0}^{\infty} g_{1}(s) d s, \int_{0}^{\infty} g_{2}(s) d s\right\}<\min \left\{\frac{2(m-1)}{2 m-1}, \frac{2(m+1)\left(E_{1}-E(0)\right)}{(2 m-1) \lambda_{1}^{2}}\right\},
$$

where $\lambda_{1}$ and $E_{1}$ are given in (4.3) and (4.4), respectively.

Next, we define the functional $G$ that helps in establishing desired results by

$$
G(\chi):=\frac{1}{2} \chi^{2}-\eta \chi^{m+1}, \quad \chi>0
$$

where $\eta$ is the constant from Lemma 2.3 .

\section{Remark 4.1}

(i) We can verify that the functional $G$ is increasing in $\left(0, \lambda_{1}\right)$, decreasing in $\left(\lambda_{1},+\infty\right)$, $G(\lambda) \rightarrow-\infty$ as $\lambda \rightarrow+\infty$, and $G$ has attains the maximum

$$
E_{1}:=G\left(\lambda_{1}\right)=\frac{m-1}{2(m+1)} \lambda_{1}^{2}
$$

at

$$
\lambda_{1}:=\left(\frac{1}{\eta(m+1)}\right)^{\frac{1}{m-1}}
$$

(ii) We observe from (3.3), Lemma 2.3, and (4.3) that

$$
\begin{aligned}
E(t) & \geq J(t) \geq \frac{1}{2} w^{2}(t)-\int_{\Omega} F(u, v) d x \\
& \geq \frac{1}{2} w^{2}(t)-\eta\left(l\|\nabla u\|_{2}^{2}+k\|\nabla v\|_{2}^{2}\right)^{\frac{m+1}{2}} \\
& \geq \frac{1}{2} w^{2}(t)-\eta w^{m+1}(t)=G(w(t)),
\end{aligned}
$$

where

$$
\begin{aligned}
w(t):= & \left(\|\Delta u\|_{2}^{2}+\|\Delta v\|_{2}^{2}+\left\|\nabla u_{t}\right\|_{2}^{2}+\left\|\nabla v_{t}\right\|_{2}^{2}+l\|\nabla u\|_{2}^{2}\right. \\
& \left.+k\|\nabla v\|_{2}^{2}+g_{1} \circ \nabla u+g_{2} \circ \nabla v\right)^{\frac{1}{2}} .
\end{aligned}
$$

Before we state and prove our main result, we need the following lemma, which is similar to a lemma from [17] to study some classes of the coupled equations.

Lemma 4.2 Assume that (A1) and (2.4) hold, $u_{0}, v_{0} \in H_{0}^{2}(\Omega)$, and $u_{1}, v_{1} \in H_{0}^{1}(\Omega)$. Let $(u, v)$ be a solution of (1.1) with initial data satisfying $E(0)<E_{1}$ and $w(0)>\lambda_{1}$, that is,

$$
\left(\left\|\Delta u_{0}\right\|_{2}^{2}+\left\|\Delta v_{0}\right\|_{2}^{2}+\left\|\nabla u_{1}\right\|_{2}^{2}+\left\|\nabla v_{1}\right\|_{2}^{2}+l\left\|\nabla u_{0}\right\|_{2}^{2}+k\left\|\nabla v_{0}\right\|_{2}^{2}\right)^{\frac{1}{2}}>\lambda_{1} .
$$


Then there exists $\lambda_{2}>\lambda_{1}$ such that, for all $t \geq 0$,

$$
w(t) \geq \lambda_{2} .
$$

Proof The proof is similar to that of Lemma 4.2 in [17].

Theorem 4.3 Suppose that (A1), (2.4), and (4.1) hold, $u_{0}, v_{0} \in H_{0}^{2}(\Omega)$, and $u_{1}, v_{1} \in H_{0}^{1}(\Omega)$. Assume further that $m>\max (p, q)$ and $I(0)<0$. Suppose that one of the following is satisfied:

(i) $E(0)<0$,

(ii) $0 \leq E(0)<E_{1}$ and $w(0)>\lambda_{1}$.

Then the solution of problem (1.1) blows up at a finite time, that is, there exists $T<+\infty$ such that

$$
\begin{aligned}
& \lim _{t \rightarrow T^{-}}\left(\left\|u_{t}\right\|_{2}^{2}+\left\|v_{t}\right\|_{2}^{2}+\|\Delta u\|_{2}^{2}+\|\Delta v\|_{2}^{2}+\left\|\nabla u_{t}\right\|_{2}^{2}+\left\|\nabla v_{t}\right\|_{2}^{2}+\|\nabla u\|_{2}^{2}+\|\nabla v\|_{2}^{2}\right. \\
& \left.\quad+\|u\|_{m+1}^{m+1}+\|v\|_{m+1}^{m+1}\right)=+\infty .
\end{aligned}
$$

Proof For case (ii), $0 \leq E(0)<E_{1}$, set

$$
H(t):=E_{2}-E(t), \quad t \geq 0
$$

where $E_{2}:=\frac{E_{1}+E(0)}{2}$. By (3.4) we see that $H^{\prime}(t) \geq 0$. Thus, we obtain

$$
H(t) \geq H(0)=E_{2}-E(0), \quad t \geq 0 .
$$

Moreover, from (4.5), (4.8), and (4.3) we see that

$$
\begin{aligned}
H(t) & =E_{2}-E(t) \\
& \leq E_{1}-\frac{1}{2} w^{2}(t)+\int_{\Omega} F(u, v) d x \\
& \leq E_{1}-\frac{1}{2} \lambda_{1}^{2}+\int_{\Omega} F(u, v) d x \\
& =-\frac{\lambda_{1}^{2}}{m+1}+\int_{\Omega} F(u, v) d x .
\end{aligned}
$$

Then, by (4.11), (4.12), and Lemma 2.2 we have

$$
0<H(0) \leq H(t) \leq \int_{\Omega} F(u, v) d x \leq c_{1}\left(\|u\|_{m+1}^{m+1}+\|v\|_{m+1}^{m+1}\right) .
$$

Let

$$
\begin{aligned}
A(t):= & H^{1-\sigma}(t)+\epsilon \int_{\Omega}\left(u u_{t}+v v_{t}\right) d x+\frac{\epsilon}{2} \int_{\Omega}\left(|\nabla u|^{2}+|\nabla v|^{2}\right) d x \\
& +\epsilon \int_{\Omega}\left(\nabla u \cdot \nabla u_{t}+\nabla v \cdot \nabla v_{t}\right) d x,
\end{aligned}
$$


where $\epsilon$ and $\sigma$ are positive constants to be specified latter. By taking the derivative of (4.14) and using Eq. (1.1) with $\gamma=\delta=1$ we get

$$
\begin{aligned}
A^{\prime}(t)= & (1-\sigma) H^{-\sigma}(t) H^{\prime}(t)+\epsilon\left(\left\|u_{t}\right\|_{2}^{2}+\left\|v_{t}\right\|_{2}^{2}\right)-\epsilon\left(\|\Delta u\|_{2}^{2}+\|\Delta v\|_{2}^{2}\right) \\
& +\epsilon\left(\left\|\nabla u_{t}\right\|_{2}^{2}+\left\|\nabla v_{t}\right\|_{2}^{2}\right)-\epsilon\left(\|\nabla u\|_{2}^{2}+\|\nabla v\|_{2}^{2}\right) \\
& +\epsilon \int_{\Omega} \int_{0}^{t} g_{1}(t-s) \nabla u(s) \cdot \nabla u(t) d s d x \\
& +\epsilon \int_{\Omega} \int_{0}^{t} g_{2}(t-s) \nabla v(s) \cdot \nabla v(t) d s d x-\epsilon \int_{\Omega}\left(u\left|u_{t}\right|^{p-1} u_{t}+v\left|v_{t}\right|^{q-1} v_{t}\right) d x \\
& +(m+1) \epsilon \int_{\Omega} F(u, v) d x .
\end{aligned}
$$

Using the Hölder and Young inequalities, we observe that

$$
\begin{aligned}
& \int_{\Omega} \int_{0}^{t} g_{1}(t-s) \nabla u(s) \cdot \nabla u(t) d s d x \\
& \quad=\int_{\Omega} \int_{0}^{t} g_{1}(t-s) \nabla u(t) \cdot(\nabla u(s)-\nabla u(t)) d s d x+\int_{0}^{t} g_{1}(t-s) d s\|\nabla u(t)\|_{2}^{2} \\
& \quad \geq-\left(g_{1} \circ \nabla u\right)+\frac{3}{4} \int_{0}^{t} g_{1}(s) d s\|\nabla u(t)\|_{2}^{2}
\end{aligned}
$$

and

$$
\int_{\Omega} \int_{0}^{t} g_{2}(t-s) \nabla v(s) \cdot \nabla v(t) d s d x \geq-\left(g_{2} \circ \nabla v\right)+\frac{3}{4} \int_{0}^{t} g_{2}(s) d s\|\nabla v(t)\|_{2}^{2} .
$$

Taking (4.16) and (4.17) into account, using (4.10) and the definition of $E(t)$ by (3.3) to substitute for $\int_{\Omega} F(u, v) d x,(4.15)$ becomes

$$
\begin{aligned}
A^{\prime}(t) \geq & (1-\sigma) H^{-\sigma}(t) H^{\prime}(t)+\epsilon a_{1}\left(\left\|u_{t}\right\|_{2}^{2}+\left\|v_{t}\right\|_{2}^{2}\right)+\epsilon a_{1}\left(\left\|\nabla u_{t}\right\|_{2}^{2}+\left\|\nabla v_{t}\right\|_{2}^{2}\right) \\
& +\epsilon a_{2}\left(\|\Delta u\|_{2}^{2}+\|\Delta v\|_{2}^{2}\right)+\epsilon a_{2}\left(g_{1} \circ \nabla u+g_{2} \circ \nabla v\right)+\epsilon a_{3}\left(\|\nabla u\|_{2}^{2}+\|\nabla v\|_{2}^{2}\right) \\
& -\epsilon \int_{\Omega}\left(u\left|u_{t}\right|^{p-1} u_{t}+v\left|v_{t}\right|^{q-1} v_{t}\right) d x+(m+1) \epsilon H(t)-(m+1) \epsilon E_{2},
\end{aligned}
$$

where

$$
\begin{aligned}
& a_{1}=\frac{m+3}{2}, \quad a_{2}=\frac{m-1}{2}, \\
& a_{3}=\frac{m-1}{2}-\frac{2 m-1}{4} \max \left\{\int_{0}^{\infty} g_{1}(s) d s, \int_{0}^{\infty} g_{2}(s) d s\right\} .
\end{aligned}
$$

By (4.1) we observe that $a_{3}>0$, and then by the definition of $w(t)$ by (4.6) we have

$$
\begin{aligned}
A^{\prime}(t) \geq & (1-\sigma) H^{-\sigma}(t) H^{\prime}(t)+\epsilon a_{1}\left(\left\|u_{t}\right\|_{2}^{2}+\left\|v_{t}\right\|_{2}^{2}\right)+\epsilon a_{3}\left(\|\Delta u\|_{2}^{2}+\|\Delta v\|_{2}^{2}\right) \\
& +\epsilon a_{3}\left(\left\|\nabla u_{t}\right\|_{2}^{2}+\left\|\nabla v_{t}\right\|_{2}^{2}\right)+\epsilon a_{3}\left(l\|\nabla u\|_{2}^{2}+k\|\nabla v\|_{2}^{2}\right)+\epsilon a_{3}\left(g_{1} \circ \nabla u+g_{2} \circ \nabla v\right) \\
& -\epsilon \int_{\Omega}\left(u\left|u_{t}\right|^{p-1} u_{t}+v\left|v_{t}\right|^{q-1} v_{t}\right) d x+(m+1) \epsilon H(t)-(m+1) \epsilon E_{2}
\end{aligned}
$$




$$
\begin{aligned}
= & (1-\sigma) H^{-\sigma}(t) H^{\prime}(t)+\epsilon a_{1}\left(\left\|u_{t}\right\|_{2}^{2}+\left\|v_{t}\right\|_{2}^{2}\right)+\epsilon a_{3} w^{2}(t) \\
& -\epsilon \int_{\Omega}\left(u\left|u_{t}\right|^{p-1} u_{t}+v\left|v_{t}\right|^{q-1} v_{t}\right) d x \\
& +(m+1) \epsilon H(t)-(m+1) \epsilon E_{2} .
\end{aligned}
$$

Since $w(t) \geq \lambda_{2}$ by $(4.8)$ and $\lambda_{2}>\lambda_{1}$ by Lemma 4.2 , we note that

$$
\begin{aligned}
a_{3} w^{2}(t)-(m+1) E_{2} & =a_{3} \frac{\lambda_{2}^{2}-\lambda_{1}^{2}}{\lambda_{2}^{2}} w^{2}(t)+a_{3} \lambda_{1}^{2} \frac{w^{2}(t)}{\lambda_{2}^{2}}-(m+1) E_{2} \\
& \geq c_{2} w^{2}(t)+c_{3}
\end{aligned}
$$

where $c_{2}=a_{3} \frac{\lambda_{2}^{2}-\lambda_{1}^{2}}{\lambda_{2}^{2}}>0$ and $c_{3}=a_{3} \lambda_{1}^{2}-(m+1) E_{2}$.

Furthermore, by the definition of $E_{1}, E_{2}=\frac{E_{1}+E(0)}{2}$ and assumption (4.1) we see that

$$
\begin{aligned}
c_{3} & =a_{3} \lambda_{1}^{2}-(m+1) E_{2} \\
& =\left(\frac{m-1}{2}-\frac{2 m-1}{4} \max \left\{\int_{0}^{\infty} g_{1}(s) d s, \int_{0}^{\infty} g_{2}(s) d s\right\}\right) \lambda_{1}^{2}-(m+1) E_{2} \\
& =\frac{(m+1)\left(E_{1}-E(0)\right)}{2}-\frac{(2 m-1) \lambda_{1}^{2}}{4} \max \left\{\int_{0}^{\infty} g_{1}(s) d s, \int_{0}^{\infty} g_{2}(s) d s\right\}>0 .
\end{aligned}
$$

Therefore, based on the above arguments, we conclude that

$$
\begin{aligned}
A^{\prime}(t) \geq & (1-\sigma) H^{-\sigma}(t) H^{\prime}(t)+\epsilon a_{1}\left(\left\|u_{t}\right\|_{2}^{2}+\left\|v_{t}\right\|_{2}^{2}\right)+\epsilon c_{2} w^{2}(t) \\
& -\epsilon \int_{\Omega}\left(u\left|u_{t}\right|^{p-1} u_{t}+v\left|v_{t}\right|^{q-1} v_{t}\right) d x+(m+1) \epsilon H(t) .
\end{aligned}
$$

To proceed further, by the Hölder and Young inequalities we have

$$
\left.\left|\int_{\Omega}\right| u_{t}\right|^{p-1} u_{t} u d x \mid \leq \frac{\delta_{1}^{p+1}}{p+1}\|u\|_{p+1}^{p+1}+\frac{p \delta_{1}^{-\frac{p+1}{p}}}{p+1}\left\|u_{t}\right\|_{p+1}^{p+1}
$$

and

$$
\left.\left|\int_{\Omega}\right| v_{t}\right|^{q-1} v_{t} v d x \mid \leq \frac{\delta_{2}^{q+1}}{q+1}\|v\|_{q+1}^{q+1}+\frac{q \delta_{2}^{-\frac{q+1}{q}}}{q+1}\left\|v_{t}\right\|_{q+1}^{q+1},
$$

where $\delta_{1}$ and $\delta_{2}$ are positive constants depending on $t$ and will be specified later.

Then, inserting the last two inequalities into (4.18), we obtain

$$
\begin{aligned}
A^{\prime}(t) \geq & (1-\sigma) H^{-\sigma}(t) H^{\prime}(t)+\epsilon a_{1}\left(\left\|u_{t}\right\|_{2}^{2}+\left\|v_{t}\right\|_{2}^{2}\right)+\epsilon c_{2} w^{2}(t) \\
& -\epsilon\left(\frac{\delta_{1}^{p+1}}{p+1}\|u\|_{p+1}^{p+1}+\frac{p \delta_{1}^{-\frac{p+1}{p}}}{p+1}\left\|u_{t}\right\|_{p+1}^{p+1}+\frac{\delta_{2}^{q+1}}{q+1}\|v\|_{q+1}^{q+1}+\frac{q \delta_{2}^{-\frac{q+1}{q}}}{q+1}\left\|v_{t}\right\|_{q+1}^{q+1}\right) \\
& +(m+1) \epsilon H(t) .
\end{aligned}
$$


At this point, choosing $\delta_{1}$ and $\delta_{2}$ such that

$$
\delta_{1}^{-\frac{p+1}{p}}=M_{1} H^{-\sigma}(t), \quad \delta_{2}^{-\frac{q+1}{q}}=M_{2} H^{-\sigma}(t)
$$

we get that

$$
\begin{aligned}
A^{\prime}(t) \geq & (1-\sigma-M \epsilon) H^{-\sigma}(t) H^{\prime}(t)+\epsilon a_{1}\left(\left\|u_{t}\right\|_{2}^{2}+\left\|v_{t}\right\|_{2}^{2}\right)+\epsilon c_{2} w^{2}(t) \\
& -\epsilon M_{1}^{-p} H^{\sigma p}(t)\|u\|_{p+1}^{p+1}-\epsilon M_{2}^{-q} H^{\sigma q}(t)\|v\|_{q+1}^{q+1}+(m+1) \epsilon H(t),
\end{aligned}
$$

where $M_{1}, M_{2}$ are positive constants, and $M=\frac{p M_{1}}{p+1}+\frac{q M_{2}}{q+1}$. It follows from (4.13) that

$$
M_{1}^{-p} H^{\sigma p}(t) \leq M_{1}^{-p} c_{1}^{\sigma p}\left(\|u\|_{m+1}^{m+1}+\|v\|_{m+1}^{m+1}\right)^{\sigma p}
$$

and

$$
M_{2}^{-q} H^{\sigma q}(t) \leq M_{2}^{-q} c_{1}^{\sigma q}\left(\|u\|_{m+1}^{m+1}+\|v\|_{m+1}^{m+1}\right)^{\sigma q}
$$

Substitution of these two inequalities into (4.19) yields

$$
\begin{aligned}
A^{\prime}(t) \geq & (1-\sigma-M \epsilon) H^{-\sigma}(t) H^{\prime}(t)+\epsilon a_{1}\left(\left\|u_{t}\right\|_{2}^{2}+\left\|v_{t}\right\|_{2}^{2}\right) \\
& +\epsilon c_{2} w^{2}(t)-\epsilon M_{1}^{-p} c_{1}^{\sigma p}\left(\|u\|_{m+1}^{m+1}+\|v\|_{m+1}^{m+1}\right)^{\sigma p}\|u\|_{p+1}^{p+1} \\
& -\epsilon M_{2}^{-q} c_{1}^{\sigma q}\left(\|u\|_{m+1}^{m+1}+\|v\|_{m+1}^{m+1}\right)^{\sigma q}\|v\|_{q+1}^{q+1}+(m+1) \epsilon H(t) .
\end{aligned}
$$

Since $p<m$ and $q<m$, we note that

$$
\begin{aligned}
& \|u\|_{p+1}^{p+1} \leq c_{4}\|u\|_{m+1}^{p+1} \leq c_{4}\left(\|u\|_{m+1}+\|v\|_{m+1}\right)^{p+1} \\
& \|v\|_{q+1}^{q+1} \leq c_{5}\|v\|_{m+1}^{q+1} \leq c_{5}\left(\|u\|_{m+1}+\|v\|_{m+1}\right)^{q+1}
\end{aligned}
$$

where $c_{4}=\operatorname{vol}(\Omega)^{\frac{m-p}{m+1}}$ and $c_{5}=\operatorname{vol}(\Omega)^{\frac{m-q}{m+1}}$. Thus,

$$
\begin{aligned}
A^{\prime}(t) \geq & (1-\sigma-M \epsilon) H^{-\sigma}(t) H^{\prime}(t)+\epsilon a_{1}\left(\left\|u_{t}\right\|_{2}^{2}+\left\|v_{t}\right\|_{2}^{2}\right)+\epsilon c_{2} w^{2}(t) \\
& -\epsilon M_{1}^{-p} c_{1}^{\sigma p} c_{4}\left(\|u\|_{m+1}^{m+1}+\|v\|_{m+1}^{m+1}\right)^{\sigma p}\left(\|u\|_{m+1}+\|v\|_{m+1}\right)^{p+1} \\
& -\epsilon M_{2}^{-q} c_{1}^{\sigma q} c_{5}\left(\|u\|_{m+1}^{m+1}+\|v\|_{m+1}^{m+1}\right)^{\sigma q}\left(\|u\|_{m+1}+\|v\|_{m+1}\right)^{q+1}+(m+1) \epsilon H(t) \\
\geq & (1-\sigma-M \epsilon) H^{-\sigma}(t) H^{\prime}(t)+\epsilon a_{1}\left(\left\|u_{t}\right\|_{2}^{2}+\left\|v_{t}\right\|_{2}^{2}\right)+\epsilon c_{2} w^{2}(t) \\
& -\epsilon M_{1}^{-p} c_{1}^{\sigma p} c_{7}\left(\|u\|_{m+1}+\|v\|_{m+1}\right)^{\sigma p(m+1)+p+1}+(m+1) \epsilon H(t) \\
& -\epsilon M_{2}^{-q} c_{1}^{\sigma q} c_{8}\left(\|u\|_{m+1}+\|v\|_{m+1}\right)^{\sigma q(m+1)+q+1}
\end{aligned}
$$

where the last inequality is derived from

$$
\begin{aligned}
& \left(\|u\|_{m+1}^{m+1}+\|v\|_{m+1}^{m+1}\right)^{\sigma p} \leq c_{6}\left(\|u\|_{m+1}+\|v\|_{m+1}\right)^{\sigma p(m+1)} \\
& \left(\|u\|_{m+1}^{m+1}+\|v\|_{m+1}^{m+1}\right)^{\sigma q} \leq c_{6}\left(\|u\|_{m+1}+\|v\|_{m+1}\right)^{\sigma q(m+1)}
\end{aligned}
$$


because of

$$
(x+y)^{\lambda} \leq c_{6}\left(x^{\lambda}+y^{\lambda}\right), \quad x, y \geq 0, \lambda>0, c_{6}>0,
$$

and the constants $c_{7}=c_{6} c_{4}$ and $c_{8}=c_{6} c_{5}$.

Now, letting

$$
0<\sigma<\min \left\{\frac{m-p}{p(m+1)}, \frac{m-q}{q(m+1)}, \frac{m-1}{2(m+1)}\right\},
$$

we have

$$
2 \leq \sigma p(m+1)+p+1 \leq m+1, \quad 2 \leq \sigma q(m+1)+q+1 \leq m+1 .
$$

Hence, by the inequality

$$
\|v\|_{m+1}^{s} \leq c(\operatorname{vol}(\Omega), m)\left(\|\nabla v\|_{2}^{2}+\|v\|_{m+1}^{m+1}\right), \quad v \in H_{0}^{1}(\Omega), 2 \leq s \leq m+1,
$$

we have

$$
\|u\|_{m+1}^{\sigma p(m+1)+p+1} \leq c_{9}\left(\|\nabla u\|_{2}^{2}+\|u\|_{m+1}^{m+1}\right)
$$

and

$$
\|v\|_{m+1}^{\sigma q(m+1)+q+1} \leq c_{10}\left(\|\nabla v\|_{2}^{2}+\|v\|_{m+1}^{m+1}\right)
$$

where $c_{9}$ and $c_{10}$ are positive constants. Taking (4.24) and (4.25) into consideration and using the definition of $w(t),(4.20)$ takes the form

$$
\begin{aligned}
A^{\prime}(t) \geq & (1-\sigma-M \epsilon) H^{-\sigma}(t) H^{\prime}(t)+\epsilon a_{1}\left(\left\|u_{t}\right\|_{2}^{2}+\left\|v_{t}\right\|_{2}^{2}\right)+\epsilon c_{2}\left(g_{1} \circ \nabla u+g_{2} \circ \nabla v\right) \\
& +\epsilon\left(c_{2} \beta-M_{1}^{-p} c_{1}^{\sigma p} c_{11}-M_{2}^{-q} c_{1}^{\sigma q} c_{12}\right)\left(\|\nabla u\|_{2}^{2}+\|\nabla v\|_{2}^{2}\right)+(m+1) \epsilon H(t) \\
& +\epsilon c_{2}\left(\|\Delta u\|_{2}^{2}+\|\Delta v\|_{2}^{2}+\left\|\nabla u_{t}\right\|_{2}^{2}+\left\|\nabla v_{t}\right\|_{2}^{2}\right) \\
& -\epsilon\left(M_{1}^{-p} c_{1}^{\sigma p} c_{11}+M_{2}^{-q} c_{1}^{\sigma q} c_{12}\right)\left(\|u\|_{m+1}^{m+1}+\|v\|_{m+1}^{m+1}\right),
\end{aligned}
$$

where $c_{11}=c_{6} \cdot c_{7} \cdot c_{9}$ and $c_{12}=c_{6} \cdot c_{8} \cdot c_{10}$.

At this moment, setting $a_{4}=\min \left\{c_{2} \beta, \frac{m+1}{2}\right\}$, decomposing $\epsilon(m+1) H(t)$ in (4.26) by

$$
\epsilon(m+1) H(t)=2 a_{4} \epsilon H(t)+\left(m+1-2 a_{4}\right) \epsilon H(t),
$$

and using (4.10) and Lemma 2.2, we obtain

$$
\begin{aligned}
A^{\prime}(t) \geq & (1-\sigma-M \epsilon) H^{-\sigma}(t) H^{\prime}(t)+\epsilon\left(a_{1}-a_{4}\right)\left(\left\|u_{t}\right\|_{2}^{2}+\left\|v_{t}\right\|_{2}^{2}\right) \\
& +\epsilon\left(c_{2}-a_{4}\right)\left(\|\Delta u\|_{2}^{2}+\|\Delta v\|_{2}^{2}+\left\|\nabla u_{t}\right\|_{2}^{2}+\left\|\nabla v_{t}\right\|_{2}^{2}\right) \\
& +\epsilon\left(c_{2} \beta-M_{1}^{-p} c_{1}^{\sigma p} c_{11}-M_{2}^{-q} c_{1}^{\sigma q} c_{12}-a_{4}\right)\left(\|\nabla u\|_{2}^{2}+\|\nabla v\|_{2}^{2}\right)
\end{aligned}
$$




$$
\begin{aligned}
& +\epsilon\left(2 a_{4} c_{0}-\left(M_{1}^{-p} c_{1}^{\sigma p} c_{11}+M_{2}^{-q} c_{1}^{\sigma q} c_{12}\right)\right)\left(\|u\|_{m+1}^{m+1}+\|v\|_{m+1}^{m+1}\right) \\
& +\epsilon\left(c_{2}-a_{4}\right)\left(g_{1} \circ \nabla u+g_{2} \circ \nabla v\right)+\left(m+1-2 a_{4}\right) \epsilon H(t) .
\end{aligned}
$$

Choosing $M_{1}$ and $M_{2}$ large enough such that

$$
\begin{aligned}
& c_{2} \beta-M_{1}^{-p} c_{1}^{\sigma p} c_{11}-M_{2}^{-q} c_{1}^{\sigma q} c_{12}-a_{4}>\frac{c_{2} \beta-a_{4}}{2}, \\
& 2 a_{4} c_{0}-\left(M_{1}^{-p} c_{1}^{\sigma p} c_{11}+M_{2}^{-q} c_{1}^{\sigma q} c_{12}\right)>a_{4} c_{0},
\end{aligned}
$$

we get

$$
\begin{aligned}
A^{\prime}(t) \geq & (1-\sigma-M \epsilon) H^{-\sigma}(t) H^{\prime}(t)+\epsilon c_{13}\left(\left\|u_{t}\right\|_{2}^{2}+\left\|v_{t}\right\|_{2}^{2}\right)+\epsilon c_{14}\left(g_{1} \circ \nabla u+g_{2} \circ \nabla v\right) \\
& +\epsilon c_{14}\left(\|\Delta u\|_{2}^{2}+\|\Delta v\|_{2}^{2}+\left\|\nabla u_{t}\right\|_{2}^{2}+\left\|\nabla v_{t}\right\|_{2}^{2}\right)+\epsilon c_{15} H(t) \\
& +\epsilon c_{16}\left(\|\nabla u\|_{2}^{2}+\|\nabla v\|_{2}^{2}\right)+\epsilon c_{17}\left(\|u\|_{m+1}^{m+1}+\|v\|_{m+1}^{m+1}\right)
\end{aligned}
$$

for some positive constants $c_{i}, i=13,14, \ldots, 17$. Once $M_{1}$ and $M_{2}$ are fixed, we pick $\epsilon>0$ small enough such that

$$
1-\sigma-M \epsilon \geq 0
$$

and

$$
\begin{aligned}
A(0)= & H^{1-\sigma}(0)+\epsilon \int_{\Omega}\left(u_{0} u_{1}+v_{0} v_{1}\right) d x+\frac{\epsilon}{2}\left(\left\|\nabla u_{0}\right\|_{2}^{2}+\|\nabla v\|_{2}^{2}\right) \\
& +\epsilon \int_{\Omega}\left(\nabla u_{0} \cdot \nabla u_{1}+\nabla v_{0} \cdot \nabla v_{1}\right) d x>0 .
\end{aligned}
$$

Thus, there exists $K>0$ such that

$$
\begin{aligned}
A^{\prime}(t) \geq & \epsilon K\left(\left\|u_{t}\right\|_{2}^{2}+\left\|v_{t}\right\|_{2}^{2}+\left\|\nabla u_{t}\right\|_{2}^{2}+\left\|\nabla v_{t}\right\|_{2}^{2}+\|\nabla u\|_{2}^{2}\right. \\
& \left.+\|\nabla v\|_{2}^{2}+\|u\|_{m+1}^{m+1}+\|v\|_{m+1}^{m+1}+H(t)\right),
\end{aligned}
$$

which, together with (4.27), implies that

$$
A(t) \geq A(0)>0, \quad t \geq 0 .
$$

On the other hand, by the Hölder and Young inequalities, (4.22), and (4.23) we have that

$$
\begin{aligned}
& \left(\int_{\Omega}\left(u_{t} u+v_{t} v\right) d x\right)^{\frac{1}{1-\sigma}} \\
& \quad \leq 2^{\frac{\sigma}{1-\sigma}}\left(\left\|u_{t}\right\|_{2}^{\frac{1}{1-\sigma}}\|u\|_{2}^{\frac{1}{1-\sigma}}+\left\|v_{t}\right\|_{2}^{\frac{1}{1-\sigma}}\|v\|_{2}^{\frac{1}{1-\sigma}}\right) \\
& \quad \leq c_{18}\left(\left\|u_{t}\right\|_{2}^{\frac{1}{1-\sigma}}\|u\|_{m+1}^{\frac{1}{1-\sigma}}+\left\|v_{t}\right\|_{2}^{\frac{1}{1-\sigma}}\|v\|_{m+1}^{\frac{1}{1-\sigma}}\right) \\
& \quad \leq c_{19}\left(\left\|u_{t}\right\|_{2}^{2}+\left\|v_{t}\right\|_{2}^{2}+\|u\|_{m+1}^{\frac{2}{1-2 \sigma}}+\|v\|_{m+1}^{\frac{2}{1-2 \sigma}}\right) \\
& \quad \leq c_{20}\left(\left\|u_{t}\right\|_{2}^{2}+\left\|v_{t}\right\|_{2}^{2}+\|\nabla u\|_{2}^{2}+\|\nabla v\|_{2}^{2}+\|u\|_{m+1}^{m+1}+\|v\|_{m+1}^{m+1}\right)
\end{aligned}
$$


and, similarly,

$$
\begin{aligned}
& \left(\int_{\Omega}\left(\nabla u_{t} \cdot \nabla u+\nabla v_{t} \cdot \nabla v\right) d x\right)^{\frac{1}{1-\sigma}} \\
& \quad \leq 2^{\frac{\sigma}{1-\sigma}}\left(\left\|\nabla u_{t}\right\|_{2}^{\frac{1}{1-\sigma}}\|\nabla u\|_{2}^{\frac{1}{1-\sigma}}+\left\|\nabla v_{t}\right\|_{2}^{\frac{1}{1-\sigma}}\|\nabla v\|_{2}^{\frac{1}{1-\sigma}}\right) \\
& \quad \leq c_{21}\left(\left\|\nabla u_{t}\right\|_{2}^{2}+\left\|\nabla v_{t}\right\|_{2}^{2}+\|\nabla u\|_{2}^{\frac{2}{1-2 \sigma}}+\|\nabla v\|_{2}^{\frac{2}{1-2 \sigma}}\right) .
\end{aligned}
$$

By using (4.9) we get

$$
\|\nabla u\|_{2}^{\frac{2}{1-2 \sigma}} \leq c^{\frac{1}{1-2 \sigma}} \leq \frac{c^{\frac{1}{1-2 \sigma}}}{H(0)} H(t)
$$

and

$$
\|\nabla v\|_{2}^{\frac{2}{1-2 \sigma}} \leq c^{\frac{1}{1-2 \sigma}} \leq \frac{c^{\frac{1}{1-2 \sigma}}}{H(0)} H(t) .
$$

Substitution of these two inequalities into (4.30) yields

$$
\left(\int_{\Omega}\left(\nabla u_{t} \cdot \nabla u+\nabla v_{t} \cdot \nabla v\right) d x\right)^{\frac{1}{1-\sigma}} \leq c_{22}\left(H(t)+\left\|\nabla u_{t}\right\|_{2}^{2}+\left\|\nabla v_{t}\right\|_{2}^{2}\right) .
$$

Similarly, we obtain

$$
\left(\|\nabla u\|_{2}^{2}+\|\nabla v\|_{2}^{2}\right)^{\frac{1}{1-\sigma}} \leq 2^{\frac{\sigma}{1-\sigma}}\left(\|\nabla u\|_{2}^{\frac{2}{1-\sigma}}+\|\nabla v\|_{2}^{\frac{2}{1-\sigma}}\right) \leq c_{23} H(t) .
$$

By using (4.29), (4.33)-(4.34), and (4.13) we get, for $t \geq 0$,

$$
\begin{aligned}
A^{\frac{1}{1-\sigma}}(t) \leq & 2^{\frac{\sigma}{1-\sigma}}\left(H(t)+\left(\int_{\Omega}\left(u u_{t}+v v_{t}\right) d x\right)^{\frac{1}{1-\sigma}}+\left(\|\nabla u\|_{2}^{2}+\|\nabla v\|_{2}^{2}\right)^{\frac{1}{1-\sigma}}\right. \\
& \left.+\left(\int_{\Omega}\left(\nabla u \cdot \nabla u_{t}+\nabla v \cdot \nabla v_{t}\right) d x\right)^{\frac{1}{1-\sigma}}\right) \\
\leq & c_{24}\left(\left\|u_{t}\right\|_{2}^{2}+\left\|v_{t}\right\|_{2}^{2}+\left\|\nabla u_{t}\right\|_{2}^{2}+\left\|\nabla v_{t}\right\|_{2}^{2}+\|\nabla u\|_{2}^{2}+\|\nabla v\|_{2}^{2}\right. \\
& \left.+\|u\|_{m+1}^{m+1}+\|v\|_{m+1}^{m+1}\right)
\end{aligned}
$$

where $c_{i}, i=18,19, \ldots, 24$, are positive constants. Combining (4.28) and (4.35), we get

$$
A^{\prime}(t) \geq c_{25} A^{\frac{1}{1-\sigma}}(t), \quad t \geq 0,
$$

where $c_{25}=\frac{\epsilon K}{c_{24}}$. Integration of $(4.36)$ over $(0, t)$ then yields

$$
A^{\frac{\sigma}{1-\sigma}}(t) \geq \frac{1}{A \frac{-\sigma}{1-\sigma}(0)-\frac{\sigma c_{25}}{1-\sigma} t}, \quad t \geq 0 .
$$

This shows that $A(t)$ blows up in finite time $T$ and

$$
T \leq \frac{1-\sigma}{\sigma c_{25} A^{\frac{\sigma}{1-\sigma}}(0)}
$$


Furthermore, we get from (4.35) that

$$
\lim _{t \rightarrow T^{-}}\left(\left\|u_{t}\right\|_{2}^{2}+\left\|v_{t}\right\|_{2}^{2}++\left\|\nabla u_{t}\right\|_{2}^{2}+\left\|\nabla v_{t}\right\|_{2}^{2}+\|\nabla u\|_{2}^{2}+\|\nabla v\|_{2}^{2}+\|u\|_{m+1}^{m+1}+\|v\|_{m+1}^{m+1}\right)=+\infty
$$

\section{Thus, the solution of problem (1.1) blows up in finite time.}

For case (i), $E(0)<0$, we set $H(t)=-E(t)$ instead of (4.10). Then, applying the same arguments as in case (ii), we have the desired result.

\section{Competing interests}

The authors declare that they have no competing interests.

\section{Authors' contributions}

The authors declare that the study was realized in collaboration with the same responsibility. All authors read and approved the final manuscript.

\section{Acknowledgements}

The authors cordially thank the anonymous referee for his (her) valuable comments and suggestions, which lead to the improvement of this paper. This work was partially supported by NNSF of China (61374089), NSF of Shanxi Province (2014011005-2), Shanxi Scholarship council of China (2013-013), and Shanxi international science and technology cooperation projects (2014081026).

Received: 15 April 2016 Accepted: 6 June 2016 Published online: 14 June 2016

\section{References}

1. Di, HF, Shang, YD: Global existence and asymptotic behavior of solutions for the double dispersive-dissipative wave equation with nonlinear damping and source terms. Bound. Value Probl. 2015, 29 (2015)

2. Shang, YD: Initial boundary value problem of equation $u_{t t}-\Delta u-\Delta u_{t}-\Delta u_{t t}=f(u)$. Acta Math. Appl. Sin. 23, 385-393 (2000)

3. Zhang, HW, Hu, QY: Existence of global weak solution and stability of a class nonlinear evolution equation. Acta Math. Sci. Ser. A 24, 329-336 (2004)

4. Xie, YQ, Zhong, CK: The existence of global attractors for a class nonlinear equation. Anal. Appl. 336, 54-69 (2007)

5. Xu, RZ, Zhao, XR, Shen, JH: Asymptotic behavior of solution for fourth order wave equation with dispersive and dissipative terms. Appl. Math. Mech. 29, 259-262 (2008)

6. Kafini, M, Messaoudi, SA: A blow-up result in a nonlinear viscoelastic problem with arbitrary positive initial energy. Dyn. Contin. Discrete Impuls. Syst., Ser. A Math. Anal. 20(6), 657-665 (2013)

7. Xie, YQ, Zhong, CK: Asymptotic behavior of a class of nonlinear evolution equation. Nonlinear Anal. 71(11), 5095-5105 (2009)

8. Carvalho, AN, Cholewa, JW: Local well posedness, asymptotic behavior and asymptotic bootstrapping for a class of semilinear evolution equation equations of the second order in time. Trans. Am. Math. Soc. 361(5), 2567-2586 (2009)

9. Sun, CY, Yang, L, Duan, JQ: Asymptotic behavior for a semilinear second order evolution equation. Trans. Am. Math. Soc. 363(11), 6085-6109 (2011)

10. Chen, CS, Wang, H, Zhu, SL: Global attractor and decay estimates of solutions to a class of nonlinear evolution equations. Math. Methods Appl. Sci. 34(5), 497-508 (2011)

11. Xu, RZ, Yang, YB: Finite time blow up for the nonlinear fourth-order dispersive-dissipative wave equation at high energy level. Int. J. Math. 23, 1250060 (2012)

12. Messaoudi, SA, Mukiawa, SE: Existence and general decay of a viscoelastic plate equation. Electron. J. Differ. Equ. 2016, 22 (2016)

13. Liang, F, Gao, HJ: Exponential energy decay and blow-up of solutions for a system of nonlinear viscoelastic wave equations with strong damping. Bound. Value Probl. 2011, 22 (2011)

14. Han, XS, Wang, MX: Global existence and blow-up of solutions for a system of nonlinear viscoelastic wave equations with damping and source. Nonlinear Anal. TMA 71, 5427-5450 (2009)

15. Messaoudi, SA, Said-Houari, B: Global nonexistence of positive initial-energy solutions of a system of nonlinear viscoelastic wave equations with damping and source terms. J. Math. Anal. Appl. 365, 277-287 (2010)

16. Said-Houari, B, Messaoudi, SA, Guesmia, A: General decay of solutions of a nonlinear system of viscoelastic wave equations. Nonlinear Differ. Equ. Appl. 18, 659-684 (2011)

17. Wu, ST: On decay and blow-up of solutions for a system of nonlinear wave equations. J. Math. Anal. Appl. 394 360-377 (2012)

18. Nakao, M: Asymptotic stability of the bounded or almost periodic solution of the wave equation with nonlinear dissipative term. J. Math. Anal. Appl. 58(2), 336-343 (1977) 